\title{
Complex Precautions: An Advance towards Secure Computing
}

\author{
KONDA HARI KRISHNA ${ }^{1}$, M. SATYANARAYANA REDDY ${ }^{2}$ \\ ${ }^{1}$ Assistant professor - Dept. Of Computer Science \& Engineering, Sri Vasavi Engineering College (Autonomous), \\ Tadepalligudem, A.P-534101. \\ ${ }^{2}$ Assistant professor - Dept. Of Computer Science \& Engineering, Sri Vasavi Engineering College (Autonomous), \\ Tadepalligudem, A.P-534101.
}

\begin{abstract}
The security of PC systems assumes a vital part in current PC frameworks. With a specific end goal to uphold high assurance levels against noxious assault, various programming apparatuses have been as of now created. Interruption Detection System has as of late turned into a warmed research subject because of its capacity of recognizing and keeping the assaults from noxious system clients. An example coordinating IDS for system security has been proposed in this paper. Numerous system security applications depend on example coordinating to separate the danger from system activity. The expansion in system speed and activity may make existing calculations to end up an execution bottleneck. In this way it is extremely important to grow quicker and more proficient example coordinating calculation so as to defeat the inconveniences on execution.
\end{abstract}

Keywords: Enemies, Effect of adversaries, Security.

\section{INTRODUCTION}

1.1 Arrange Security: Network and PC security is basic to the money related soundness of each association. In the course of recent years, Internet-empowered business, or e-business, has definitely enhanced productivity and income development. E-business applications, for example, e-trade, production network administration, and remote get to permit organizations to streamline forms, bring down working expenses, and increment consumer loyalty. Such applications require mission-basic systems that suit voice, video, and information activity, and these systems must be adaptable to bolster expanding quantities of clients and the requirement for more noteworthy limit and execution. Be that as it may, as systems empower increasingly applications and are accessible to increasingly clients, they turn out to be perpetually powerless against a more extensive scope of security dangers. To battle those dangers and guarantee that e-business exchanges are not traded off, security innovation must assume a noteworthy part in today's systems.

1.2 Need: Security occurrences are ascending at a disturbing rate each year. As the multifaceted nature of the dangers expands, so do the efforts to establish safety required to secure systems. Server farm administrators, arrange executives, and other server farm experts need to grasp the essentials of security keeping in mind the end goal to securely convey and oversee organizes today.

1.3 Destinations: over the long haul, increasingly new innovation will be produced to encourage enhance the proficiency of business and interchanges. In the meantime, leaps forward in innovation will give considerably more noteworthy system security, subsequently, more prominent bit of brain to work in bleeding edge business situations. Given that ventures remain focused of this developing innovation, and in addition the most recent security dangers and threats, the advantages of systems will unquestionably exceed the dangers.

1.4 Significance of Network Security: To ensure organization resources: One of the essential objectives of PC and system security is the assurance of organization data that is housed on an organization's PCs and systems. To pick up an upper hand: Developing and keeping up compelling efforts to establish safety can give an association an upper hand over its opposition. Arrange security is especially critical in the field of Internet money related administrations and e-trade.

\section{ENEMIES OF NETWORK SECURITY}

2.1 Hackers: This nonspecific and frequently over-romanticized term applies to PC fans who enjoy accessing other individuals' PCs or systems.

2.2 Unconscious Staff: As representatives concentrate on their particular occupation obligations, they regularly ignore standard system security rules. Like basic secret key, utilization of Virus affecting CD/DVD and so forth.

2.3 Snoops: Employees known as "snoops" share in corporate undercover work, increasing unapproved access to private information keeping in mind the end goal to give contenders generally difficult to reach data. 


\section{EFFECT OF ENEMIES}

3.1 Infections: Viruses are the most broadly known security dangers, since they regularly gather broad squeeze scope Viruses are PC programs that are composed by naughty software engineers and are intended to reproduce themselves and taint PCs when activated by a particular occasion. A system can be contaminated by an infection just if the infection enters the system through an outside source-regularly through a tainted floppy plate or a document downloaded from the Internet. When one PC on the system gets to be contaminated, alternate PCs on the system are exceedingly helpless to getting the infection.

3.2 Trojan stallion Programs: Trojan steed projects, or trojans, are conveyance vehicles for ruinous code. Trojans give off an impression of being innocuous or helpful programming projects, for example, PC amusements, however they are really foes in camouflage. Trojans can erase information, mail duplicates of themselves to email address records, and open up PCs to extra assaults. Trojans can be contracted just by duplicating the trojan stallion program to a framework, by means of a circle, downloading from the Internet, or opening an email connection. Neither trojans nor infections can be spread through an email message itself - they are spread just through email connections.

3.3 Vandals: Web destinations have woken up through the improvement of such programming applications as ActiveX and Java Applets. These gadgets empower liveliness and other enhancements to run, making Web destinations more appealing and intuitive. Be that as it may, the simplicity with which these applications can be downloaded and run has given another vehicle to dispensing harm. A vandal is a product application or applet that causes annihilation of changing degrees. A vandal can demolish only a solitary record or a noteworthy parcel of a PC framework.

3.4 Assaults: Innumerable sorts of system assaults have been recorded, and they are normally characterized in three general classifications: Reconnaissance assaults, Access assaults and Denial of administration (DoS) assaults. Observation assaults are basically data gathering exercises by which programmers gather information that is utilized to later bargain systems. For the most part, programming instruments, for example, sniffers and scanners, are utilized to outline organize assets and endeavour potential shortcomings in the focused-on systems, hosts, and applications.

Get to assaults are directed to adventure vulnerabilities in such system regions as confirmation administrations and File Transfer Protocol (FTP) usefulness keeping in mind the end goal to pick up section to email records, databases, and other secret information. DoS assaults counteract access to part or the greater part of a PC framework. They are typically accomplished by sending a lot of cluttered or generally unmanageable information to a machine that is associated with a corporate system or the Internet, blocking authentic activity from overcoming. Significantly more malignant is a Distributed Denial of Service assault (DDoS) in which the assailant bargains different machines or has.

3.5 Information Interception: Data transmitted through a system can be liable to block attempt by unapproved parties. The culprits may listen in on correspondences or even change the information bundles being transmitted. Culprits can utilize different techniques to capture the information IP satirizing.

3.6 Social Engineering: Social designing is the inexorably predominant demonstration of acquiring secret system security data through non-specialized means.

3.7 Spam: Spam is the generally utilized term for spontaneous electronic mail or the activity of broadcasting spontaneous promoting messages through email. Spam is generally safe, yet it can be an aggravation, taking up the beneficiary's chance and storage room.

\section{SECURITY TOOLS}

After the potential wellsprings of dangers and the sorts of harm that can happen have been distinguished, putting the correct security approaches and defends set up turns out to be much simpler. Associations have a broad selection of innovations, going from hostile to infection programming bundles to devoted system security equipment, for example, firewalls and interruption identification frameworks, to give assurance to all territories of the system.

4.1 Against infection Packages: Virus assurance programming is bundled with most PCs and can counter most infection dangers if the product is frequently upgraded and effectively kept up. The counter infection industry depends on an incomprehensible system of clients to give early notices of new infections, so remedies can be produced and dispersed rapidly. With a great many new infections being created each month, it is crucial that the infection database is stayed up with the latest.

The infection database is the record held by the counter infection bundle that helps it to recognize known infections when they endeavour to strike. Respectable against infection programming sellers will distribute the most recent antitoxins on their Web destinations, and the product can provoke clients to occasionally gather new information. Arrange security approach ought to stipulate that all PCs on the system are stayed up with the latest and, in a perfect world, are all ensured by the same hostile to infection bundle - if just to keep support and redesign expenses to a base. It is additionally vital to overhaul the product itself all the time. Infection creators frequently make moving beyond the counter infection bundles their first need. 
4.2 Security Policies: When setting up a system, whether it is a neighbourhood (LAN), virtual LAN (VLAN), or wide region arrange (WAN), it is vital to at first set the essential security approaches. Security strategies are principles that are electronically modified and put away inside security hardware to control such regions as get to privileges. The arrangements that are actualized ought to control who has admittance to which ranges of the system and how unapproved clients will be kept from entering confined territories.

The individual or gathering of individuals who police and keep up the system and its security must have admittance to each range of the system. Once your approaches are set, personality techniques and innovations must be utilized to help decidedly validate and check clients and their get to benefits. Ensuring that specific zones of the system are "secret word secured"- just available by those with specific passwords - is the least difficult and most regular approach to guarantee that exclusive the individuals who have authorization can enter a specific part of the system.

The brilliant guidelines, or approaches, for passwords are:

- Change passwords routinely

- Make passwords as aimless as could be expected under the circumstances

- Never disclose passwords to anybody until leaving the Company.

Computerized testaments or open key endorsements are the electronic counterparts of driver's licenses or international IDs, and are issued by assigned Certificate Authorities (CAs). Computerized declarations are frequently utilized for recognizable proof when setting up secure passages through the Internet, for example, in virtual private systems administration (VPN).

4.3 Firewalls: A firewall is an equipment or programming arrangement executed inside the system framework to implement an association's security approaches by limiting access to particular system assets. In the physical security similarity, a firewall is the proportional to an entryway bolt on an edge entryway or on a way to a room within the building - it allows just approved clients, for example, those with a key or get to card, to enter. Firewall innovation is even accessible in variants appropriate for home utilize. The firewall makes a defensive layer between the system and the outside world. As a result, the firewall repeats the system at the purpose of section so it can get and transmit approved information immediately. Nonetheless, it has worked in channels that can deny unapproved or conceivably unsafe material from entering the genuine framework. It additionally logs an endeavoured interruption and reports it to the system chairmen.

4.4 Encryption: Encryption innovation guarantees that messages can't be caught or read by anybody other than the approved beneficiary. Encryption is generally conveyed to secure information that is transported over an open system and utilizations progressed scientific calculations to "scramble" messages and their connections.

4.5 Interruption Detection: A system-based interruption recognition framework (IDS) gives all day and all night arrange reconnaissance. An IDS investigates parcel information streams inside a system, looking for unapproved movement, for example, assaults by programmers, and empowering clients to react to security breaks before frameworks are traded off. At the point when unapproved action is recognized, the IDS can send cautions to an administration support with subtle elements of the movement and can regularly arrange different frameworks, for example, switches, to remove the unapproved sessions. In the physical similarity, an IDS is identical to a camcorder and movement sensor; distinguishing unapproved or suspicious action and working with computerized reaction frameworks, for example, watch gatekeepers, to stop the action.

\section{CONCLUSION}

Over the long haul, increasingly new innovation will be produced to advance enhance the productivity of business and correspondences. In the meantime, leaps forward in innovation will give significantly more noteworthy system security, along these lines, more prominent bit of psyche to work in forefront business situations. Given that ventures remain focused of this developing innovation, and in addition the most recent security dangers and perils, the advantages of systems will definitely exceed the dangers.

\section{REFERENCES}

1. http://en.wikipedia.org/wiki/Network_security

2. http://www.interhack.net/pubs/network-security/

3. http://e-articles.info/e/s/s/Network-security/

4. ijns.femto.com.tw/contents/ijns-v10-n1/ijns-v10-n1.html

5. pnbiit.com/download/JulSep09.pdf 


\section{INFORMATION ABOUT AUTHORS:}

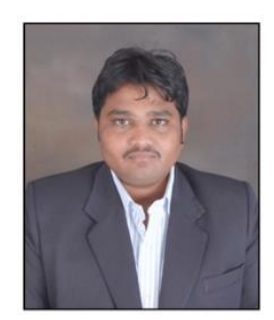

1. KONDA HARI KRISHNA, received his M.TECH in computer science from Jawaharlal Nehru Technological University, Kakinada, A.P and pursuing Ph.D in LINGAYA's Vidyapeeth University, Faridabad. He is currently working as an Asst.Prof in CSE Department of Sri Vasavi Engineering College(A), Tadepalligudem, West Godavari, A.P. He published different Research Papers in Various International Journals of Reputed and His Research Area is Improvement of Network Lifetime using Clustering and Dynamic Topology Methods in WSN. He is a good researcher \& who has worked mostly on Wireless Sensor networks, Network security, Data mining, Data analytics \& cloud computing.

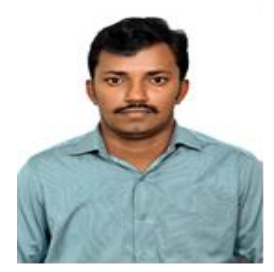

2. SATYANARAYANAREDDY MARRI, received his M.TECH in Computer Science and Engineering from Acharya Nagarjuna University Campus, Guntur, A.P and pursuing Ph.D in Jntu Ananthapur University, Anantapur. He is currently working as an Asst.Prof in CSE Department of Sri Vasavi Engineering College(A), Tadepalligudem, West Godavari, A.P. He published different Research Papers in Various International Journals of Reputed and His Research Area is Computer Networks, Network Security \& Cloud Computing. 